\title{
DEVELOPMENT OF CYBER-PHYSICAL SYSTEM FOR LASER-BASED CONTROLLED CLOUD SEEDING
}

\author{
ANKUSH RAI, JAGADEESH KANNAN R \\ School of Computing Science and Engineering, VIT University, Chennai, Tamil Nadu, India. Email: ankushressci@gmail.com
}

Received: 28 December 2016, Revised and Accepted: 10 May 2017

\section{ABSTRACT}

Objective: This research encompasses of developing a cyber-physical system, i.e., a system which can control the physical process of cloud formation by utilizing the light detection and ranging technology to modulate its frequency while directed on aerosol cloud to control the movement of cloud, formation, and precipitation in a test-bed setting.

Methods: This involves utilization of advanced algorithms, image processing techniques to model cloud behavior, and artificial intelligence to control the laser frequency modulation and pulse count, directed or incidence angle to understand the response between laser effects on aerosol cloud.

Results and Discussion: It will enable studying the detailed effects of laser-induced cloud nucleation and also in modeling the cloud behavior with respect to the feedback loop between induced affect through lasers upon the aerosol cloud.

Keywords: Computing technique, Modelling of cloud seeding, Feedback control of cyber physical system.

(C) 2017 The Authors. Published by Innovare Academic Sciences Pvt Ltd. This is an open access article under the CC BY license (http://creativecommons. org/licenses/by/4. 0/) DOI: http://dx.doi.org/10.22159/ajpcr.2017.v10s1.19643

\section{INTRODUCTION}

The last decade has witnessed the emergence of a new cloud seeding technique based on laser-induced water condensation. This has acquired a form of new research status for potentially utilizing ultrashort laser pulses for cloud seeding [1-6]. The observation of the phenomenon in various conditions by several groups is now well established, and some basic processes at play are identified [7-14]. The challenges that are being addressed in the current pre-proposal are as follows:

a. The first challenge lies in understanding the full complexity of the different pathways contributing to this mechanism which constitutes the most challenging question ahead in this field. Both the aerosol microphysics and the plasma photochemistry have to provide a deeper view and understanding of laser-generated particles from their nucleation to their ultimate evolution [15-18].

b. The second challenge ahead regards with the development of a controlled method for the atmospheric seeding of clouds. Assessing its fine-tuned parametric calibration on a sufficiently large scale requires automation and further knowledge on the efficiency of laserinduced condensation in various atmospheric conditions, to enable realistic modeling of the observed particle nucleation and growth and allow determining the cloud types, regions, and conditions, in which precipitation enhancement could be expected [19-23].

Thus, the project proposes to address the above-mentioned challenges by studying and developing a cyber-physical system (CPS) for controlling light detection and ranging (LIDAR)-induced cloud seeding as platforms of software and hardware, respectively, accounting for modeling the climate data and surveying local demand for rain. This automated command system for cloud seeding using artificial intelligence will achieve the controlled and calculated enhancement of artificial rain. It will be developed and completed based on the technique of CPS, bringing together three operations on the level of the laboratory testbed, province, and cities. Here in this study we presents utilizing new technologies of computing and modeling technique, spatial-temporal databases, GPS, and state-of-the-art algorithms of artificial intelligence in integration with meteorological products (such as radar, satellite, weather station database) to gauge cloud nucleation of cloud in order to achieve the following:

a. controlled cloud seeding,

b. accurate prediction of artificial rainfall,

c. automate modeling of such multimodal data, and

d. controlling/issuing the real-time instructions and

e. command the LIDAR system for rain enhancement

Thereby, operating cloud seeding through a computationally controlled CPS.

This research work focuses on the system's architectural outlines of design, configuration, and functional utility of software, modelers for understanding aerosol microphysics in relation with the plasma photochemistry to realize the goal of enhanced understanding of artificial cloud seeding process.

\section{METHODS}

\section{Architecture for CPS-based cloud seeding}

Fig. 1 illustrates the proposed framework. Its relative impacts of selected observing systems are assimilated for the control experiment using various configurations in terms of their fractional contributions to the reduction of a global measure of 24-hrs forecast error. It combines geographical specific data of wind, temperature, surface pressure, satellite images, and images of clouds extracted terrestrially with respect to the verifying historical data of tropospheric specific regional areas for subsequent computation and analysis. Fig. 2 shows the framework for extracting the convective cloud functional flow for the forecast algorithm namely Dynamic Boolean Network (DBN). There are three main threads: The echo tops forecast, the precipitation, and the precipitation phase. The image data are in $1 \mathrm{~km}$ resolution, with 5-minute update rates, and 0-to-2-hr forecast loops. The algorithm steps include the fundamental line and convective weather interest detections using functional template correlation and region analysis; our secondary interest lies in the detections using thresholding and region size sorting on convective and non-convective elements and a rule-based precedence ordering where the primitive images are used to assemble the final weather classification image. Multiscale tracking module takes the line (envelope) and cell sets of track vectors from 


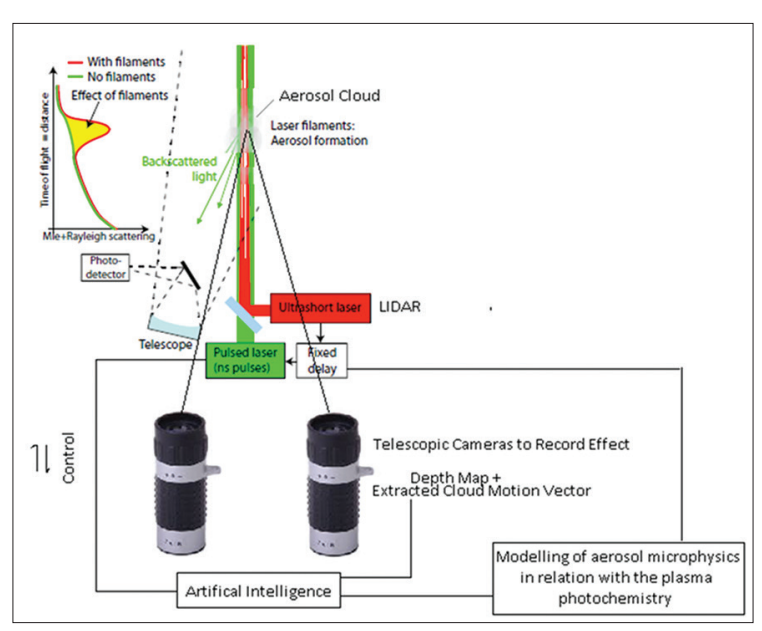

Fig. 1: Overview of the proposed cyber-physical system for controlled cloud seeding through light detection and ranging

(LIDAR) system and simultaneous modeling of aerosol microphysics which is aided by the data extracted from threedimensional cloud motion vectors after processing it through computer vision algorithms. Thereafter, forming the correlation from the processed data with the data extracted from LIDAR regarding plasma photochemistry with the help of artificial intelligence

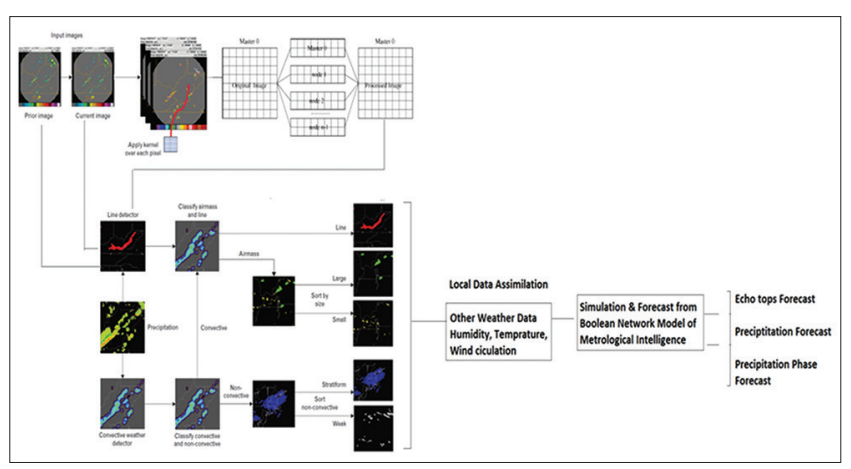

Fig. 2: Block diagram of the processing mechanism of the proposed framework

each radar and sorts them according to weather type, providing the appropriate motion to each area of weather.

Here, we intend to use our own newly mathematically modeled coactivated Boolean networks for machine learning algorithm. Unlike the current neural network, the DBN is modeled on much more deeper level. The biological neuron consists of several networks of microtubules which enable each neuron to response based on the data type or make it data driven. Thus, the current DBN which we are inclined to use will enable our CPS system to model the feature sets by enabling the check for conditional existence of other multiple-dependent conditions called coactivation. This coactivation network will be employed for the computation as it is topologically complex in several ways, and we intend to use it on our research with the combination of evolving Boolean networks.

The DBN element can be represented as $r$ items, for which there is a sequence of $\mathrm{m}$ number of frames given by $\mathrm{X} \in\left\{\mathrm{X}_{\mathrm{X}_{0}}{ }^{(1)}, \mathrm{X}_{\mathrm{X}_{0}}{ }^{(2)}, \ldots, \mathrm{X}_{\mathrm{X}_{0}}{ }^{(\mathrm{n})}\right\}$. Here, $X$ is the set of possible values of a frame. A frame could be a short video segment, a short sequence of image blocks. Content frames may overlap spatially, temporally, or both. Here, overlapping time of windows is 2 seconds long and starts every $185 \mathrm{~ms}$; with overlap of $15 / 16$ being used as frames. Suppose that C, B, and Y are matrix of filtered output, $\mathrm{Y}$ is the matrix of filters for stimulant variable and response variable for each $\mathrm{X}$, such that $\mathrm{C}=\mathrm{XBY}$. Then, $\mathrm{C}$ is a superframe of $\mathrm{B}$. The length of a frame $\mathrm{S}$ is equivalent to the total number of frames in it and is denoted by $|\mathrm{S}|$.

Now,

$$
X_{X_{0}}=\left[\begin{array}{ccc}
\mathrm{X}_{\mathrm{x}_{0}}{ }^{(1)} & \mathrm{L} & 0 \\
\mathrm{M} & 0 & \mathrm{M} \\
0 & \mathrm{~L} & \mathrm{X}_{\mathrm{x}_{0}}(\mathrm{r})
\end{array}\right], X_{X_{0}}(j)=\left[\begin{array}{c}
1 \\
0 \\
\vdots \\
1
\end{array}\right], j=1,2, \ldots r
$$

where $\mathrm{X}_{\mathrm{X}_{0}}$ is the covariance map from $\mathrm{X}$ which asserts to the association formed between the frames $\mathrm{S}(\mathrm{t})$ with that of stimulant and response variable. $\mathrm{X}_{\mathrm{i}}$ is the position of input record. $\mathrm{C}_{1}$ is the cluster value which contains various values from 1 to $l$.

Now, at each step, we calculate:

$$
\begin{gathered}
S(t)=\sum_{i=-\infty}^{+\infty} \sum_{k=1}^{N_{S C}} \mathrm{C}_{l i} S_{l}\left(t-i_{S}\right) \\
S_{l}(t)=\prod(t) e^{j 2 \pi f_{1} t} \\
\prod(t)=\left\{\begin{array}{c}
1,0<t \leq T_{S} \\
0, t \leq 0, t>T_{S}
\end{array}\right.
\end{gathered}
$$

Where $C_{1 i}$ is the $i^{\text {th }}$ information symbol at the $\mathrm{l}^{\text {th }}$ subcarrier (when output of one iteration is propagated to the input of the other), $\mathrm{T}_{\mathrm{s}}$ is the symbol period, $\mathrm{S}$ is the waveform for the $\mathrm{l}^{\text {th }}$ subcarrier, $\mathrm{N}_{\mathrm{sc}}$ is the number of subcarriers (number of matching iterations), $\mathrm{f}_{1}$ is the frequency of the subcarrier, and $\pi(t)$ is the pulse shaping function. Following this process, to complete the dataset in all records, the dynamics of the equation for a computational job using Boolean networks is computed as shown below: At time $t$, standard deviation is requested by micro devices.

$$
\delta_{\mathrm{i}}(\mathrm{x}, \mathrm{y})=\frac{\sum\left(\mathrm{I}_{\mathrm{xy}}^{\mathrm{i}}(\mathrm{t})-\overline{\mathrm{I}_{\mathrm{xy}}^{\mathrm{i}}(\mathrm{t})}\right)^{2}}{\mathrm{~T}}
$$

where $\mathrm{I}_{\mathrm{xy}}^{\mathrm{i}}(\mathrm{t})$ is the consequent frame with the location of each pixel value in the form of $(x, y)$ for the frame at time $t, I_{x y}^{i}(t)$ averaged over information of all $\mathrm{I}_{\mathrm{xy}}^{\mathrm{i}}(\mathrm{t})$ value for time t. Hence, STD for the frame comprising object $\left(\delta_{\mathrm{i}}^{0}\right)$ and its multiscale object region $\left(\delta_{\mathrm{i}}^{\mathrm{s}}\right)$ can be mapped as the quantitative information about its trajectory in continuous frame sequence can be derived from:

$$
\mathrm{s}_{\mathrm{i}}=\sum\left(\mathrm{I}_{\mathrm{xy}}(\mathrm{t})-\mathrm{I}_{\mathrm{xy}}(\mathrm{t}-1)\right)^{2}
$$

Now, we need to derive the symmetry of the object and its multiscale object region to optimize the classification process using the attributebased level adaptive algorithm $[24,25]$.

Where symmetry breakdown allows us to ease the identification problem of the object by looking for the intercorrelation between symmetry of the object and the symmetry of its multiscale object region [26]. Hence, the relationship between it can be learned in one shot for object classification, which is given as: 


$$
x(i, p) \leftarrow\left(\sum_{t=t(i, j)+1}^{t(i, j+1)-1} F_{j}(t)\right)-z(i, j)-w(i)
$$

here, $x(i, p)$ is an indicator to the event that the solution is in state $i$ during the $p_{\text {th }}$ phase of feature instance and $n_{i}$ is the number of phases of state i. Thus, forming a dynamic sequence, the nodal degree distribution was fat-tailed with high-degree hub nodes to be located in presented polymorph neural network using sequence of information to excite the necessary regions and access the information in an associative form. This enables the machine not only to learn but also to embark the cross-relationship between various data for prediction or simulation-based logical conclusion; herein, the processing is done over neural net-based shell environment [27]. Computationally, this topology was embedded parsimoniously in terms of the connection distance between coactivated nodes and the framework with which the weather intelligence shall be implemented is shown in Fig. 2. Most connections or edges were separated by short sequence of excitatory data, significantly shorter than random networks; with $\mathrm{P}<10-^{3}$ in the permutation test. Relatively few edges were long distance, and these were often interhemispheric projections between bilaterally homotopic regions where $14 \%$ of longest connections (defined as top 10 percentile) were homotopic; significantly more than random.

Furthermore, note that the rich cluster concentrates most of the activations, whereas the periphery and particularly the default-mode network concentrate the deactivations (Fig. 3). Edges represent the top 1 percentile of most consistently reported activation and deactivations (no directions shown for clarity purposes). Edges can be seen spanning across different modules. Although the network cost is usually found to be overall low, as measured by the distance of connections, the network topology still managed to balance integration and segregation between all topological artificial neural regions: The clustering of the network threshold at sparse levels is much higher than random, while retaining a similar path length. In all these respects, the organization of the coactivation network is convergent with properties of a comparable functional connectivity network generated from resting-state of excitatory sequences. As known from prior study, and reproduced here, a recognition state polymorph neural networks for feature extraction and encoding of it for both the gray-scaled and negative images (which is an example of small world encoding), with fat-tailed degree distributions and parsimonious distance distributions.

This allows the wiring of the learned neurons to be plastic (left, which rigidly affinity to particular sequence of data and exhibits the constant state once excited) and the elastic neurons (right, which returns to its initial state; mainly used for transfer of sequence from one network point to other). The spot and arrow above each receptor represent excitation that stimulates the pixel receptor. The sequence of numerical response units is generated by the elastic and plastic neurons, which in turn helps create responses to spot intensity of 2.0. For the coactivation network, it is possible to assign functional as well as anatomical labels to the modules using Boolean networks. To do this, we will consider the high-level behavioral domains used which describe each contrast in the primary literature: Action, cognition, interaction, perception, and interoception. We then labeled each edge according to the domain most frequently causing coactivation of the corresponding pair of regions. In the occipital module, the highest proportion of intramodular modeling is set aside, as once modeled, the polymorph neural network has proven its evolutionary nature and self-modeling in dynamic scenario in previous studies corresponded to coactivation by perception (39\%) and the other domains coactivated less than $20 \%$ each; similarly, in the default mode module, other domains each accounted for less than $21 \%$; whereas, in the central module, $62 \%$ of intramodular edges were coactivated by action. Thus, it seems reasonable to say that the proposed research has firm prominence with its scope fulfillment and will be relatively specialized for action, the occipital module for vision-based perception. Action and cognition tasks in regard with weather prediction accounted for approximately the same proportion of intramodular edges in the other software AI modules (34\% and 38\%, respectively), and therefore, we described it as specialized for such modeling of executive functions.

\section{RESULTS AND DISCUSSION}

As in the CPS controlled cloud chamber, we watched that the upgrade of the LIDAR-induced cloud motion by the two-axial laser is enough to induce airborne cloud seeding, modifying cloud motion vectors and induced condensation (Fig. 4). The above-depicted model of water particles dictates quantitatively affirmed this subjective contention. In view of high-perceivability conditions and air-mass back directions, we considered an underlying cloud vector for circulation. In the first place, the watched impact diminishes when the foundation signal from LIDAR expands, i.e. when more water droplets are accessible for discontinuous dispersion. In addition, the effects could vary from laboratory test-bed to remote mainland or urban land, which were likewise considered, without influencing the outcome subjectively. The perceivability gave the water molecules focus, which was equivalent to $126 \mathrm{~mm}^{-3}$. Somewhere around 8 and 400 ppm for each dispersed water molecule were considered, with refractive lists in the range 1.23-1.45 generally experienced in fogs. Regardless of the possibility that an overestimation of the filament (97) and of corresponding diameter across (196 mm) was considered in the study, we found that induced dispersion could expand the Mie backscattering coefficient by at generally $0.1-0.2 \%$. In this way, induced localization of cloud motion vectors gives the predominant commitment to the CPS-based cloud seeding.

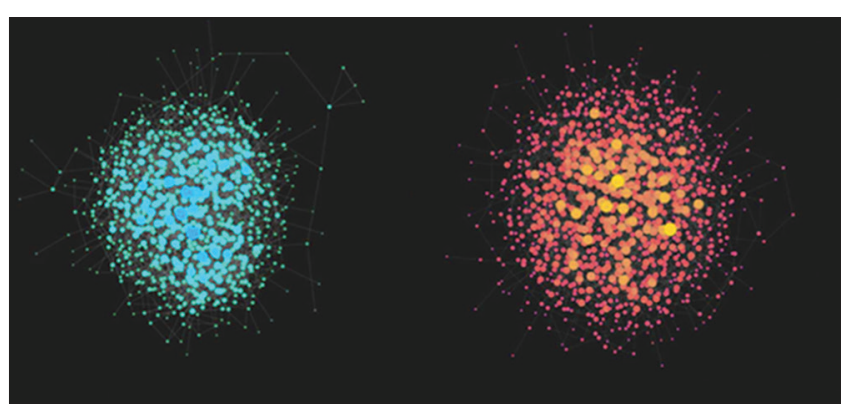

Fig. 3: Topological illustration of the functional network of coactivation in Dynamic Boolean Network for classification of features. Here, the layout of the features corresponding to the minimum spanning tree is used to locate nodes in relation to their topological proximity to each other. Different modules are coded by color proportional to their weighted degree. In addition, those nodes in anatomical space are colored based upon its proportionality to activations and deactivations

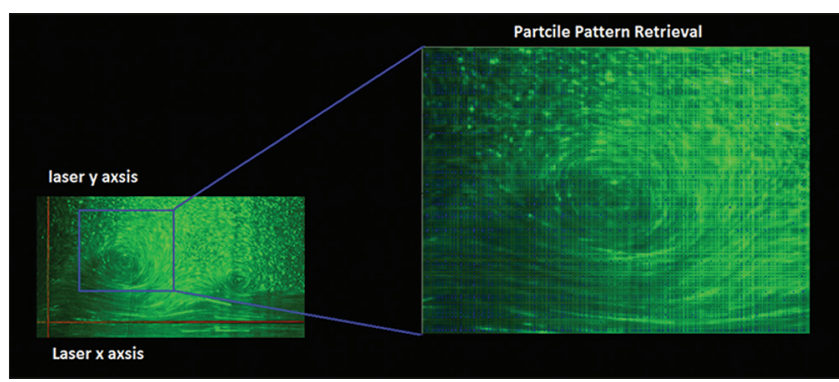

Fig. 4: The two cyber-physical system controlled axial lasers acting on the cloud particle under closed condition forming vortices and cloud motion vectors for cloud transportation and changing its morphology by controlling the entropy of particles, dynamics, and temperature 


\section{CONCLUSION}

In this study, we have shown that CPS-guided lasers could be employed for cloud seeding in closed surrounding. The modified cloud motion vectors generated by pulses of ultra-short laser can help in accelerated condensation of water by increasing the entropy. This result would help in understanding the effects in open atmosphere and triggering cloud formation in ARD regions of India. This helps us in extracting the essential parameters to fine tune the complete cloud seeding process, and thus, the outcome of the research could be operated at lower cost than that of cloud seeding offered by chemical rockets.

\section{REFERENCES}

1. Becker A, Aközbek N, Vijayalakshmi K, Oral E, Bowden CM, Chin SL. Intensity clamping and re-focusing of intense femtosecond laser pulses in nitrogen molecular gas. Appl Phys B Lasers Opt 2001;73:287.

2. Daigle JF, Becker AJ, Hosseini S, Wang TJ, Kamali Y, Roy G, et al. Intensity clamping measurement of laser filaments in air at 400 and 800 nm. Phys Rev A 2010;82:023405.

3. Kasparian J, Sauerbrey R, Chin SL. The critical laser intensity of selfguided light filaments in air. Appl Phys B Lasers Opt 2000;71:877.

4. Béjot P, Kasparian J, Henin S, Loriot V, Vieillard T, Hertz E, et al. Higher-order Kerr terms allow ionization-free filamentation in gases. Phys Rev Lett 2010;104:103903.

5. Béjot P, Hertz E, Lavorel B, Kasparian J, Wolf JP, Faucher O. Transition from plasma- to Kerr-driven laser filamentation. Phys Rev Lett 2011;106:243902.

6. Shen YR. The Principles of Nonlinear Optics. New York: WileyInterscience; 1984. p. 303-24.

7. Méjean G, Kasparian J, Yu J, Salmon E, Frey S, Wolf JP, et al. Multifilamentation transmission through fog. Phys Rev E 2005;72:026611.

8. Henin S, Petit Y, Kasparian J, Wolf JP, Jochmann A, Kraft SD, et al. Saturation of the filament density of ultrashort intense laser pulses in air. Appl Phys B 2010;100:77.

9. Petit Y, Henin S, Nakaema WM, Béjot P, Jochmann A, Kraft SD. 1-J white-light continuum from 100-TW laser pulses. Phys Rev A 2011;83:013805

10. Rodriguez M, Bourayou R, Méjean G, Kasparian J, Yu J, Salmon E, et al. Kilometer-range nonlinear propagation of femtosecond laser pulses. Phys Rev E Stat Nonlin Soft Matter Phys 2004;69:036607.

11. Méchain G, Couairon A, André YB, D’Amico C, Franco M, Prade B, et al. Long-range self-channeling of infrared laser pulses in air: A new propagation regime without ionization. Appl Phys B 2004;79:379.

12. Wille H, Rodriguez M, Kasparian J, Mondelain D, Yu J, Mysyrowicz A, et al. Teramobile: A mobile femtosecond-terawatt laser and detection system. Eur Phys J Appl Phys 2002;20:183-90.

13. Strickland D, Mourou G. Compression of amplified chirped optical pulses. Opt Commun 1985;56:219.

14. Liu W, Théberge F, Arévalo E, Gravel JF, Becker A, Chin SL. Experiment and simulations on the energy reservoir effect in femtosecond light filaments. Opt Lett 2005;30:2602-4.

15. Courvoisier F, Boutou V, Kasparian J, Salmon E, Méjean G, Yu J, et al. Light filaments transmitted through clouds. Appl Phys Lett 2003;83:213-5.

16. Kolesik M, Moloney JV. Self-healing femtosecond light filaments. Opt Lett 2004;29:590-2.

17. Skupin S, Bergé L, Peschel U, Luderer F. Interaction of femtosecond light filaments with obscurants in aerosols. Phys Rev Lett 2004;93:023901.

18. Méchain G, Méjean G, Ackermann R, Rohwetter P, André YB, Kasparian J, et al. Propagation of FS-TW laser filaments in adverse atmospheric conditions. Appl Phys B 2005;80:785-9.

19. Ackermann R, Méjean G, Kasparian J, Yu J, Salmon E, Wolf JP. Laser filaments generated and transmitted in highly turbulent air. Opt Lett 2006;31:86-8.

20. Salamé R, Lascoux N, Salmon E, Kasparian J, Wolf JP. Propagation of laser filaments through an extended turbulent region. Appl Phys Lett 2007;91:171106.

21. Kasparian J, Rodriguez M, Méjean G, Yu J, Salmon E, Wille H, et al. White-light filaments for atmospheric analysis. Science 2003;301(5629):61-4.

22. Kasparian J, Wolf JP. Physics and applications of atmospheric nonlinear optics and filamentation. Opt Express 2008;16(1):466-93.

23. Kamali Y, Daigle JF, Théberge F, Châteauneuf M, Azarm A, Chen Y, et al. Remote sensing of trace methane using mobile femtosecond laser system of T\&T lab. Opt Commun 2009;282:2062-5.

24. Rai A. Attribute based level adaptive thresholding algorithm for object extraction. J Adv Robot 2015;1(2):64-8.

25. Rai A. Attribute based level adaptive thresholding algorithm (ABLATA) for image compression and transmission. J Math Comput Sci 2014;12:211-8.

26. Rai A, Ramanathan S. Distributed learning in networked controlled cyber physical system. Int J Pharm Technol 2016;8(3):18537-46.

27. Rai A. Shell implementation of neural net over the UNIX environment for file management: A step towards automated operating system. J Oper Syst Dev Trends 2014;1(2):10-4. 